\title{
Exposure to high levels of magnesium disrupts bone mineralization in vitro and in vivo
}

\author{
Wenxiang Chu ${ }^{1,2 \#}$, Tao Li $^{1,3 \#}$, Gaozhi Jia ${ }^{4}$, Yongyun Chang ${ }^{1}$, Zhiqing Liu ${ }^{1}$, Jia Pei ${ }^{4}$, Degang Yu ${ }^{1}$, Zanjing Zhai ${ }^{1}$ \\ ${ }^{1}$ Shanghai Key Laboratory of Orthopaedic Implants, Department of Orthopaedic Surgery, Shanghai Ninth People's Hospital, Shanghai Jiao Tong \\ University School of Medicine, Shanghai, China; ${ }^{2}$ Department of Orthopedic Surgery, Changzheng Hospital, Second Military Medical University, \\ Shanghai, China; ${ }^{3}$ Department of Orthopaedics, Xinhua Hospital affiliated to Shanghai Jiaotong University School of Medicine, Shanghai, China; \\ ${ }^{4}$ National Engineering Research Center of Light Alloy Net Forming \& State Key Laboratory of Metal Matrix Composite, School of Materials \\ Science and Engineering, Shanghai Jiao Tong University, Shanghai, China \\ Contributions: (I) Conception and design: Z Zhai, W Chu; (II) Administrative support: D Yu, T Li; (III) Provision of study materials or patients: J Pei, \\ G Jia; (IV) Collection and assembly of data: W Chu, Y Chang, Z Liu; (V) Data analysis and interpretation: W Chu, T Li, Z Zhai; (VI) Manuscript \\ writing: All authors; (VII) Final approval of manuscript: All authors. \\ "These authors contributed equally to this work. \\ Correspondence to: Zanjing Zhai, Jia Pei. No.639, Zhizaoju Road, Huangpu District, Shanghai, China. Email: zanjing_zhai@163.com, jpei@sjtu.edu.cn; \\ Degang Yu. No.800, Dongchuan Road, Minhang District, Shanghai, China. Email: ydg163@126.com.
}

Background: The removal of permanent internal fixation devices by secondary surgery could be avoided if these devices were made of degradable magnesium and magnesium alloys. Before such implants can be used clinically, however, the biological effect of magnesium exposure on surrounding bone must be evaluated. Previous studies have focused on bone formation; few have examined the effects of magnesium on the bone quality that affect many biomechanical properties.

Methods: Using bone quality parameters, we analyzed in vivo changes in bone properties and biomechanics after exposure to locally high levels of magnesium.

Results: Local bone mineralization was significantly disrupted following exposure to a porous rod of pure magnesium. Normal crystal formation and crystallinity were inhibited and the mineral-to-matrix ratio decreased. These results were consistent with those of in vitro experiments, in which high levels of magnesium inhibited mineral deposition by mesenchymal stem cells (MSCs) but increased alkaline phosphatase (ALP) expression. The same mineralization inhibition was observed around magnesium implants via micro-computerized tomography (micro-CT) and von Kossa staining. Such reduced bone quality around degrading magnesium rods could negatively impact bone biomechanics.

Conclusions: This study showed that exposure to the local high magnesium levels that arise from rapidly degrading magnesium devices may significantly disrupt bone mineralization and negatively impact bone biomechanics.

Keywords: Magnesium; degradable implants; bone quality; mineralization

Submitted Feb 25, 2020. Accepted for publication Aug 28, 2020.

doi: $10.21037 / \mathrm{atm}-20-1921$

View this article at: http://dx.doi.org/10.21037/atm-20-1921

\section{Introduction}

Internal fixation devices made of magnesium and magnesium alloys are promising in the field of orthopedics due to their safety (1), their appropriate mechanical properties on fixation, their biodegradability, and their elastic modulus that resembles human bone $(2,3)$, as well as osteogenesis (4). Many commercial magnesium-based internal fixation devices have been designed to address a variety of clinical presentations $(5,6)$. However, before these novel magnesium alloy devices can transform the clinical 
landscape of orthopedics, a comprehensive understanding of their effect on bone is required. Unfortunately, findings as to these effects remain controversial; there is inconsistency from cellular studies (7-9), in vivo studies $(10-12)$, and even some small-scale clinical trials $(1,13,14)$. An objective assessment with a comprehensive and effective evaluation system is urgently needed to improve products and address any problems that arise throughout the clinical transformation of magnesium-based devices.

At present, bone tissue staining and bone density as assessed by micro-computerized tomography (micro-CT) are the primary methods to measure the impact of magnesium on bone. However, these methods usually omit two important indicators of bone strength: bone composition and bone structure (15). The National Institutes of Health proposes that assessing bone quality can alleviate this issue, as bone quality encompasses all of the properties that affect fracture resistance, including material composition, bone microstructure, and bone modeling and remodeling $(16,17)$. The concept of bone quality highlights the influence of factors other than bone density on bone strength and can more comprehensively predict and evaluate a bone's mechanical function, including its resistance to fracture. In this study, we incorporated bone quality into the existing evaluation system to comprehensively assess the biological effects of magnesium on surrounding bone and to provide a reference for the design and further development of magnesium-based internal fixation devices. We present the following study in accordance with the ARRIVE reporting checklist (available at http://dx.doi.org/10.21037/ atm-20-1921).

\section{Methods}

\section{Response of cellular osteogenic fate to magnesium stimulation in vitro}

Animal experiments were performed under a project license (HKDL[2018]293) granted by the Animal Ethics Committee of the Shanghai Ninth People's Hospital affiliated with the Shanghai Jiao Tong University School of Medicine, in compliance with Shanghai Ninth People's Hospital affiliated with the Shanghai Jiao Tong University School of Medicine guidelines for the care and use of animals. Bone marrow $(2 \mathrm{~mL})$ was aspirated from each volunteer and cultured as reported in previous studies (18). Third-generation human bone marrow stromal osteoprogenitor cells (hBMSCs) at a concentration of $1 \times 10^{5} / \mathrm{mL}$ were inoculated into 24 -well plates and incubated at $37{ }^{\circ} \mathrm{C}, 5 \% \mathrm{CO}_{2}$. After 1 day of incubation, the standard medium was replaced with osteogenic medium (standard medium $+0.1 \mathrm{mM}$ dexamethasone, $50 \mathrm{mM}$ ascorbic acid and $10 \mathrm{mM} \beta$-glycerophosphate sodium) containing either $0,3,6$, or $9 \mathrm{mM} \mathrm{MgCl}$. This medium was changed every 2 days. After 7 days, the cultures were stained with alkaline phosphatase (ALP). First, adherent cells were fixed with $4 \%$ paraformaldehyde for 15 minutes at room temperature and then immersed in ALP dye solution (Shanghai Hongqiao, Shanghai) at $37^{\circ} \mathrm{C}$ for 1 hour before observation. An ALP viability assay was performed with an ALP microplate test kit (Nanjing Jiancheng Bioengineering Institute, China) following the manufacturer's instructions. After a 21-day induction, adherent cells were fixed with $4 \%$ paraformaldehyde and stained with $1 \%$ alizarin red solution (Sigma-Aldrich, USA) at $37{ }^{\circ} \mathrm{C}$ for 45 minutes. The cells were then rinsed again with phosphate-buffered saline (PBS) to remove non-specific staining and a semiquantitative analysis for mineralized nodules dissolved in 10\% cetylpyridinium chloride (C9002-25G; Sigma-Aldrich, USA) was performed by measuring absorbance at $562 \mathrm{~nm}$.

\section{Preparation and characterization of porous magnesium rods}

\section{Preparation of magnesium-based scaffolds}

Porous scaffolds composed of pure magnesium alloy (99.99\%, Henan Yuhang Metal Materials Co. Ltd., China) were fabricated using the template replication method; details can be found in our earlier publication (19). Briefly, a pure magnesium ingot was placed on top of a tightly packed template of $\mathrm{NaCl}$ (particle size $=400 \mu \mathrm{m}$ ) in a steel mold and heated to a molten state. After the molten metal permeated the $\mathrm{NaCl}$ template, it was solidified. Subsequently, the green compact of magnesium and the packed template were immersed twice in copious amounts of ultrapure water to completely remove the $\mathrm{NaCl}$ template and obtain the open porous magnesium scaffolds. These were blow-dried in a stream of nitrogen gas and stored in a vacuum. The porous magnesium rods ( $4 \mathrm{~mm} \Phi \times 8 \mathrm{~mm}$ ) were cut into 4 equal parts $(2 \mathrm{~mm} \Phi \times 8 \mathrm{~mm})$ for implantation.

\section{Characterization of scaffolds}

The microscopic morphology and surface chemical composition of the magnesium scaffolds was measured with a scanning electron microscope (SEM) (JSM 7600F, Japan) and energy dispersive X-ray spectroscopy (EDS) (Oxford, UK). The scaffolds were coated in a thin layer of gold with a sputter coater (SHINKKU VD MSP-1S, Japan) before SEM examination to 
improve conductivity. The Archimedean method was used to characterize the scaffolds' accurate volumes, and their porosity value was derived from their relative volume.

\section{In vitro degradation}

The magnesium scaffolds were subjected to an in vitro immersion test, which involved incubation in $45 \mathrm{~mL}$ Dulbecco's Modified Eagle Medium (Gibco, USA) under cell culture conditions $\left(37^{\circ} \mathrm{C}, 5 \% \mathrm{CO}_{2}\right.$, and $95 \%$ humidity) for up to 4 weeks. The concentration of $\mathrm{Mg}^{2+}$ released into the medium was analyzed using an inductively coupled plasma atomic emission spectrometer (ICP-AES, iCAP6300, USA) and the $\mathrm{pH}$ value was measured with a $\mathrm{pH}$ meter (FE20, Mettler Toledo, Switzerland). The average corrosion rate was calculated by the weight loss postimmersion of three independent scaffold samples.

\section{Animal experiments}

All animal experiments were approved by the Animal Ethics Committee of the Shanghai Ninth People's Hospital affiliated with the Shanghai Jiao Tong University School of Medicine. Included in the experiment were $24 \mathrm{New}$ Zealand rabbits (18 weeks old, $2.5 \pm 0.3 \mathrm{~kg}$ ). The rabbits were equally divided into two groups: a negative control group (NC group) and an experimental group ( $\mathrm{Mg}$ group). All animals were fully anesthetized before surgery. After the animals were shaved and disinfected, the right femoral condyle was surgically exposed. A defect $3 \mathrm{~mm} \Phi \times 8 \mathrm{~mm}$ deep was made in the right femoral condyle with an electric drill. A prepared magnesium rod was implanted in the defects of the experimental group animals. No material was implanted in the defect of the $\mathrm{NC}$ animals. Two months post surgery, 6 rabbits from each group were sacrificed, and 4 months post surgery, the rest of the rabbits in each group were sacrificed. Healthy bone from the same point on the contralateral limb was taken as positive control (PC group).

\section{Energy-dispersive spectroscopy and energy-dispersive spectroscopy mapping analysis}

Bone centered on the defect area with an inner diameter of $5 \mathrm{~mm}$ was removed by circumcision, and the specimens were placed in a lyophilizer for 48 hours to remove any moisture. Energy-dispersive spectroscopy (EDS) and EDS mapping were performed at areas peripheral to the defect in the lyophilized samples. Scanning electron microscopeenergy dispersive spectroscopy (SEM-EDS) (Tescan VEGA
II, Czech) was used to determine the chemical composition on the surface of the annular bone around the edge of the defect. Surface elements on the same area, including calcium, phosphorus, and magnesium, were also measured by EDS mapping analysis.

\section{Bone quality analysis}

\section{Raman spectroscopy}

Raman spectroscopy analysis was performed on the bone near the defect area using an InVia Raman (Ranishaw, USA) equipped with a $785 \mathrm{~nm}$ laser emitter (100× objective). The spectral range included a Raman wavelength of $800-1,800 \mathrm{~cm}^{-1}$. Data were normalized by multi-point baseline correction and curve fitting was applied with WiRE 4.0 software (Ranishaw, USA).

\section{Fourier transform infrared spectroscopy}

Following Raman spectroscopy analysis, the samples were milled and mixed with potassium bromide $(\mathrm{KBr})$ for infrared (IR) measurement. Attenuated total reflectance-Fourier transform infrared spectroscopy (ATR-FTIR) (Spotlight 400, PerkineElmer, USA) was performed at a resolution of $4 \mathrm{~cm}^{-1}$ over a range of $400-1,800 \mathrm{~cm}^{-1}$. Spectra were baseline corrected using the instrument's software (Spectrum) and integrated areas of bands were calculated using OriginPro 9.1 software (OriginLab Corporation, Massachusetts, USA).

\section{Thermogravimetric analysis}

The remaining samples were heated from 25 to $700{ }^{\circ} \mathrm{C}$ at a rate of $10^{\circ} \mathrm{C} / \mathrm{min}$ using a TA SDT Q600 TGA (TA Instruments, USA) and the percentage weight residue was taken as the mineral ratio in the bone.

\section{Micro-computed tomography}

Micro-CT was used to evaluate bone structural parameters, including bone mineral density (BMD), bone volume fraction (BV/TV), bone surface to volume ratio (BS/ $\mathrm{BV})$, trabecular number (Tb.N), trabecular thickness (Tb.Th), and trabecular spacing (Tb.Sp), as well as the extent of magnesium rod degradation. The rabbit femoral condyles were placed in a Micro-CT81 scanner (SCANCO MEDICAL AG, Brüttisellen, Switzerland) with a $50 \mathrm{kV}$ voltage, $200 \mu \mathrm{A}$ current, and a resolution of $5.8 \mu \mathrm{m} /$ pixel. The regions of interest (ROIs) consisted of a $5 \mathrm{~mm}$ diameter column centered on the defect area $(5 \mathrm{~mm} \Phi \times 8 \mathrm{~mm})$ and a $1 \mathrm{~mm}$ ring-shaped column around the defect area. 


\section{Mechanical analysis}

A mechanical testing machine (H25K-S, Hounsfield Test Equipment, UK) connected to a $2.5 \mathrm{~mm}$ diameter indentation rod was used to test $5 \mathrm{~mm} \Phi \times 8 \mathrm{~mm}$ cylindrical cancellous bone centered on the defect area. The rod was loaded into the center of the samples with a $1,000 \mathrm{~N}$ load cell at a rate of $10 \mathrm{~mm} / \mathrm{min}$ to record maximum load displacement and elastic modulus (MPa).

\section{Histological evaluation}

The femoral condyles were fixed in paraformaldehyde for 1 week before dehydration with a series of graded ethanol solutions. The specimens were then embedded in methyl methacrylate (MMA) and cut in transverse sections of approximately $200 \mathrm{~mm}$ thickness. The sections were further ground down and polished to $50 \mathrm{~mm}$ thickness for von Kossa staining and van Gieson staining. Image-Pro Plus 6.0 (Media Cybernetics, Maryland, USA) was used to measure the area of mineralized bone following von Kossa staining.

\section{Statistical analysis}

The data were analyzed using SPSS 24.0 software (SPSS Inc., Chicago, IL, USA). Groups comparison was performed with the Student's t-test and multiple comparisons were performed by means of one-way analysis of variance (ANOVA), followed by the least significant difference test. A significant difference between groups was represented by $\mathrm{P}<0.05$. All data are presented as the mean \pm standard deviation.

\section{Results}

High levels of magnesium promote early osteogenesis but inbibit late mineral deposition in bBMSCs in vitro

As the magnesium ion concentration gradient increased, the expression of ALP by hBMSCs increased in a concentration gradient-dependent manner (Figure 1A,B). Conversely, bone mineral deposition showed gradient-dependent inhibition (Figure 1A,C).

\section{Characterization of porous magnesium rods and in vitro degradation}

The process of magnesium scaffold fabrication is illustrated in Figure 2A. SEM examination confirmed that the pore structure of the scaffolds generally followed the morphology of the $\mathrm{NaCl}$ microparticle template, showing a spatially uniform open porous structure that was spherical in shape (Figure 2B). The porous structure consisted of macropores with a diameter of approximately $400 \mu \mathrm{m}$ and interconnecting micropores with diameters of 150-200 $\mu \mathrm{m}$. The interconnected pores were homogeneously distributed along the smooth walls of the macropores. The porosity of the magnesium scaffold was approximately $71 \%$. The chemical composition of the scaffold surfaces was further examined by EDS, which clearly reflected surface element mapping of magnesium (Figure 2B).

The degradation of magnesium scaffolds was determined by the electrochemical corrosion reaction of magnesium in physiological solutions, which releases $\mathrm{Mg}^{2+}, \mathrm{OH}^{-}$and $\mathrm{H}_{2}$ gas as the primary degradation products. The in vitro degradation profile of the magnesium scaffolds while immersed in cell culture medium for 4 weeks was reflected by changes in $\mathrm{pH}$ value and the quantity of $\mathrm{Mg}^{2+}$ released (Figure 2C,D). Throughout the immersion period, the $\mathrm{pH}$ of the medium rapidly increased to more than 8.3 within 3 days and then continued to slowly increase to approximately 8.5-8.6 over the next 25 days. The cumulative amount of $\mathrm{Mg}^{2+}$ released from the scaffolds showed a gradually increasing trend, reaching approximately $57 \mathrm{mg}$ by the end of 4 weeks. According to ASTM G31-72, the average corrosion rate of the magnesium scaffolds during 4 weeks of immersion was approximately $0.55 \mathrm{~mm} /$ year.

\section{Bone mineral phase and magnesium distribution around rods}

EDS and EDS mapping suggested that the bone samples were rich in carbon and oxygen and that calcium and phosphorus distribution was widespread in every group. No magnesium distribution was detected in the positive or negative control groups. Magnesium was detectable in the bone surrounding the magnesium rods at 2 months post surgery and was not detected at 4 months post surgery (Figure 3).

\section{Exogenous magnesium disrupts bone mineralization}

Raman spectroscopy analysis was used to detect the mineralization activity of bone near the defect area (Figure 4A,B,C,D). The $v_{1}\left(\mathrm{PO}_{4}^{-3}\right)$ wavelength peak of healthy bone occurs at $961.0 \pm 0.2 \mathrm{~cm}^{-1}$. The $v_{1}\left(\mathrm{PO}_{4}^{-3}\right)$ 

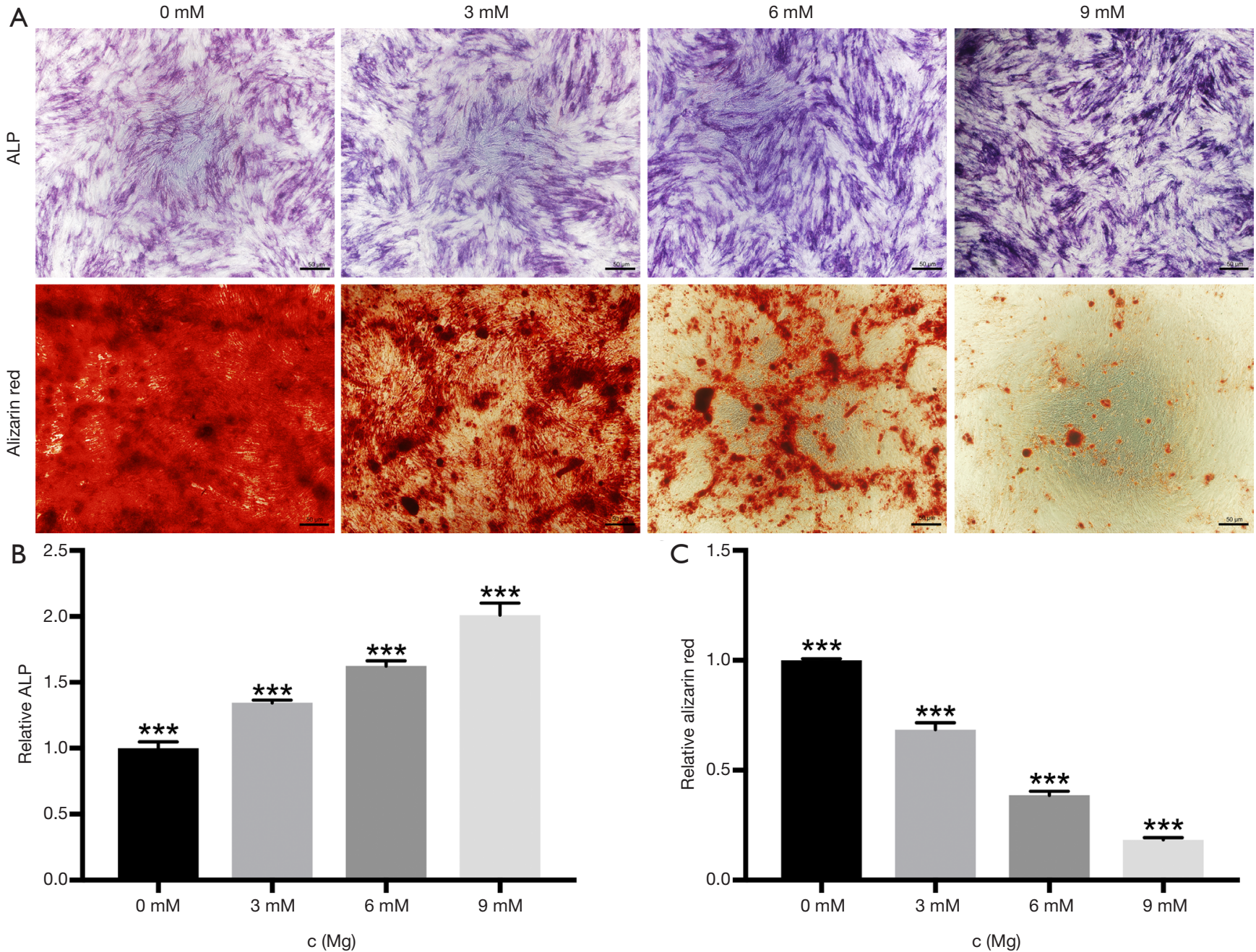

Figure 1 Effects of magnesium on early osteogenic differentiation and late bone mineral deposition in human bone marrow stromal osteoprogenitor cells (hBMSCs). (A) Representative alkaline phosphatase (ALP) and alizarin red staining of hBMSCs after stimulation for 7 days and 21 days in osteogenic medium supplemented with $0,3,6$, and $9 \mathrm{mM} \mathrm{MgCl}_{2}$. (B, C) A semi-quantitative comparison of ALP expression at 7 days and alizarin red staining at 21 days between groups with different magnesium concentrations. The asterisks in the bar graphs stand for " $\mathrm{P}<0.01$ " between this group and the other three groups.

wavelength peak positions in the NC group $\left(960.1 \pm 0.6 \mathrm{~cm}^{-1}\right)$ and the $\mathrm{Mg}$ group $\left(960.0 \pm 0.5 \mathrm{~cm}^{-1}\right)$ were significantly lower than that in the $\mathrm{PC}$ group $(\mathrm{P}<0.01)$ at 2 months post surgery, but there was no significant difference between the $\mathrm{NC}$ and $\mathrm{Mg}$ groups $(\mathrm{P}>0.05)$. There was no significant difference between the NC group $\left(961.2 \pm 0.3 \mathrm{~cm}^{-1}\right)$ and the PC group in terms of $v_{1}\left(\mathrm{PO}_{4}^{-3}\right)$ wavelength peak position $(\mathrm{P}>0.05)$ at 4 months post surgery, but the wavelength peak position in the $\mathrm{Mg}$ group $\left(960.2 \pm 1.4 \mathrm{~cm}^{-1}\right)$ was significantly lower than that in the NC group $(\mathrm{P}<0.05)$ (Figure $4 B)$. The full width at half-maximum of the $v_{1}\left(\mathrm{PO}_{4}^{-3}\right)$ peak (FWHM) in healthy bone was measured as $16.7 \pm 0.6 \mathrm{~cm}^{-1}$. The FWHM in the
PC group was significantly lower than that in the NC group $\left(18.4 \pm 1.3 \mathrm{~cm}^{-1} ; \mathrm{P}<0.01\right)$ and the $\mathrm{Mg}$ group $\left(18.0 \pm 1.0 \mathrm{~cm}^{-1}\right.$; $\mathrm{P}<0.05)$ at 2 months post surgery. There was no significant difference in FWHM between the NC group $\left(17.3 \pm 1.3 \mathrm{~cm}^{-1}\right)$ and the PC group 4 months post surgery, while the FWHM was significantly higher in the $\mathrm{Mg}$ group $\left(24.0 \pm 5.9 \mathrm{~cm}^{-1}\right)$ than in the $\mathrm{NC}$ group $(\mathrm{P}<0.01)$ and the $\mathrm{PC}$ group $(\mathrm{P}<0.01)$ (Figure $4 C$ ). The mineral-to-matrix ratio, as measured by $v_{1}\left(\mathrm{PO}_{4}^{-3}\right)$ peak value to type I collagen peak value, in the $\mathrm{Mg}$ group (2.2 \pm 1.4$)$ was significantly lower than that in the $\mathrm{NC}$ group $(4.6 \pm 1.3 ; \mathrm{P}<0.01)$ and in the $\mathrm{PC}$ group $(5.3 \pm 1.5$; $\mathrm{P}<0.01)$ at 2 months post surgery. The same was true at 


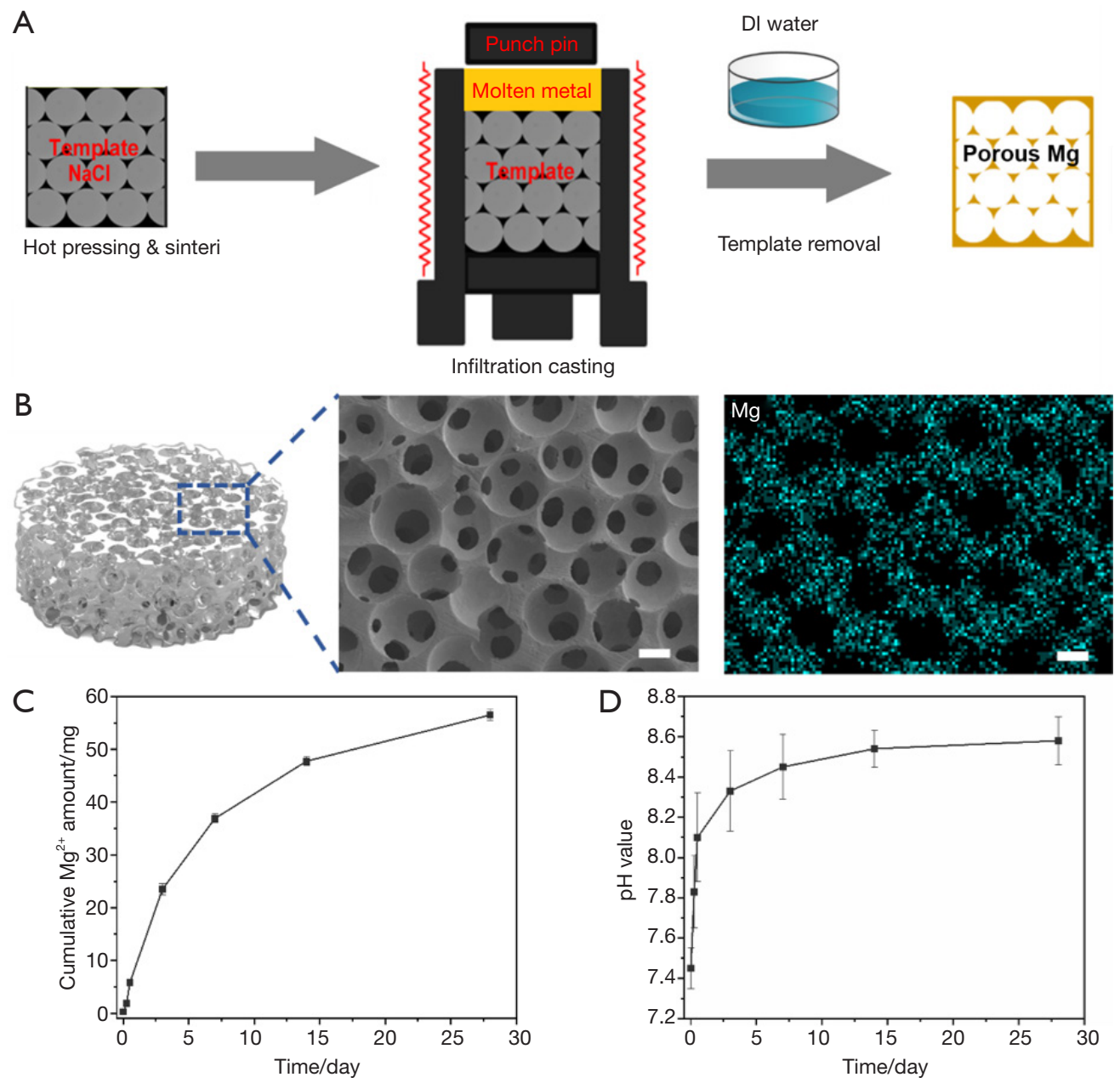

Figure 2 Preparation and characterization of porous magnesium rods. (A) Schematic diagram showing the process used to fabricate the porous magnesium scaffolds. (B) Scanning electron microscope (SEM) morphology and energy dispersive X-ray spectroscopy (EDS) element mapping of magnesium scaffold samples; the scale bar represents $200 \mu \mathrm{m}$. In vitro degradation profile of (C) the cumulative amount of $\mathrm{Mg}^{2+}$ released and (D) changes in $\mathrm{pH}$ during immersion of the magnesium scaffolds in cell culture medium for up to 4 weeks.

4 months post-surgery $(1.2 \pm 2.2$ vs. $5.0 \pm 3.1, \mathrm{P}<0.05$ and $1.2 \pm 2.2$ vs. $5.3 \pm 1.5, \mathrm{P}<0.01$, respectively) (Figure $4 D$ ).

FTIR was used to compare relative amounts of organic compounds in the bone of each group (Figure $4 E$ and $F$ ). After normalizing to that of healthy bone, the area under the curve (AUC) in the wavelength range of 1,200 to $1,550 \mathrm{~cm}^{-1}$ in the $\mathrm{NC}$ group $(2.0 \pm 0.4 ; \mathrm{P}<0.01)$ and the $\mathrm{Mg}$ group $(1.8 \pm 0.1 ; \mathrm{P}<0.05)$ was significantly higher than that in the PC group at 2 months post surgery, while there was no significant difference between the $\mathrm{NC}$ and $\mathrm{Mg}$ groups ( $\mathrm{P}>0.05)$. At 4 months post surgery, however, the AUC was significantly higher in the $\mathrm{Mg}$ group $(1.7 \pm 0.3)$ than in the NC group $(1.1 \pm 0.3 ; \mathrm{P}<0.05)$ (Figure $4 F)$.

Thermogravimetric analysis was used to quantitatively assess bone mineral content (Figure $4 G$ ). After being heated to $700{ }^{\circ} \mathrm{C}$, the residual healthy cancellous bone accounted for $47.0 \% \pm 2.1 \%$ of its dry weight. This value was $38.6 \% \pm 2.3 \%$ in the NC group at 2 months post surgery, which was significantly lower than that in the PC group $(\mathrm{P}<0.01)$. The value increased to $43.4 \% \pm 1.9 \%$ at 4 months post-surgery, and there was no significant difference compared with the value in healthy bone $(\mathrm{P}>0.05)$. The residual accounted for $23.8 \% \pm 2.8 \%$ in the $\mathrm{Mg}$ group at 2 months post surgery, which was significantly lower than that in both the $\mathrm{NC}$ and the $\mathrm{PC}$ groups $(\mathrm{P}<0.01)$. The value had recovered by 4 months post surgery, climbing to $34.3 \% \pm 3.7 \%$, but it remained significantly lower than in the $\mathrm{NC}$ and $\mathrm{PC}$ groups $(\mathrm{P}<0.01)$. 


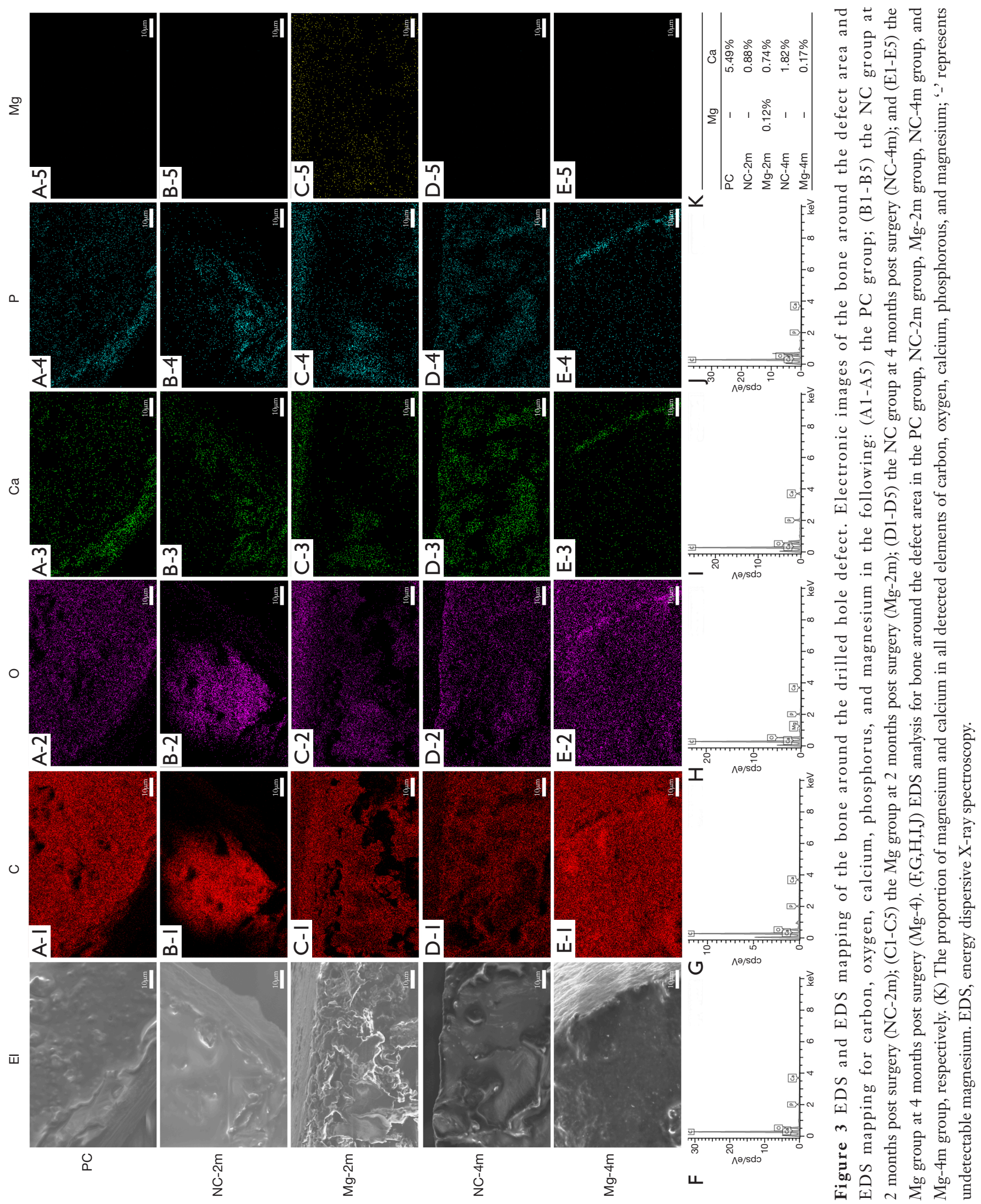


A
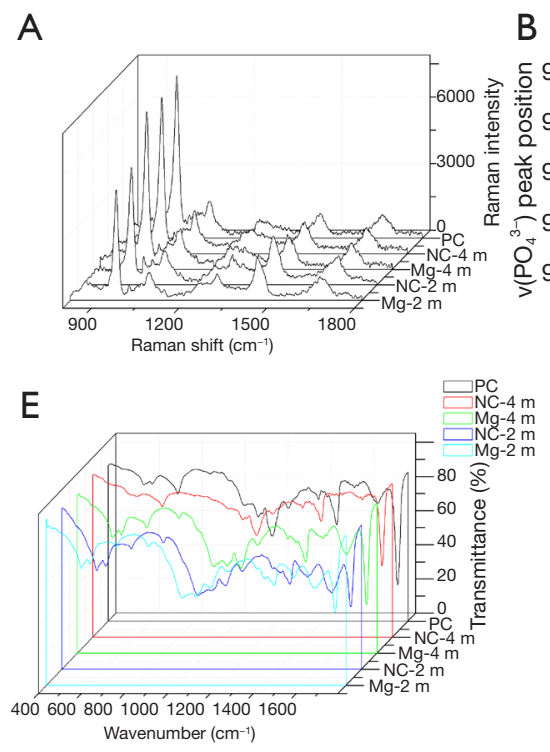

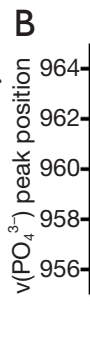

\section{$\infty^{\infty}$}

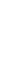

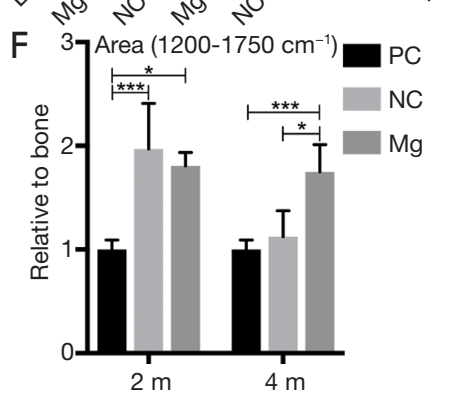

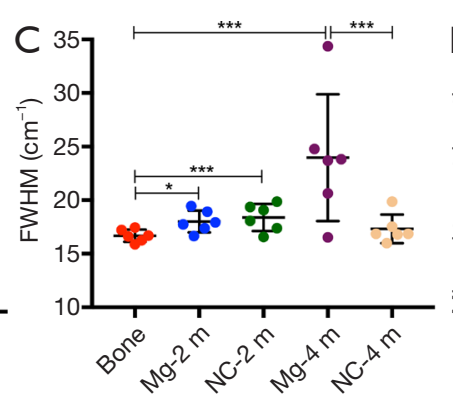

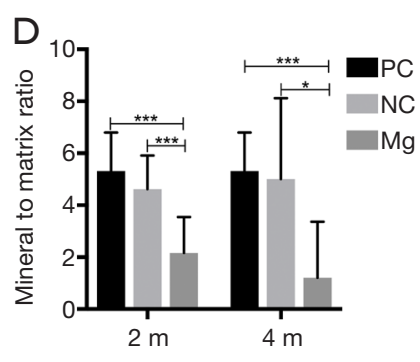

G

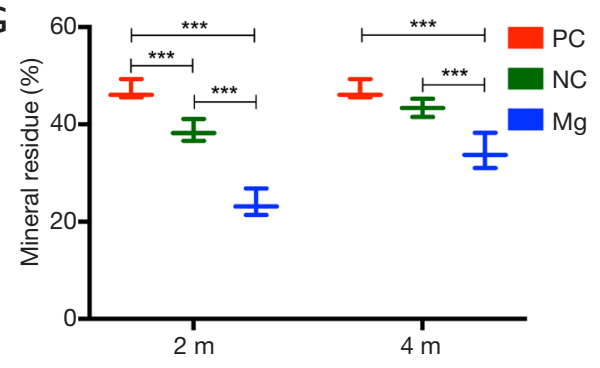

Figure 4 Effects of high levels of magnesium on bone quality in vivo. (A) Representative Raman spectra of bone close to the defect area in the PC and NC-2m groups; the Mg-2m group; the NC-4m group; and the Mg-4m group. (B) Comparison of Raman wavelength position of the $v 1\left(\mathrm{PO}_{4}{ }^{3-}\right)$ peak in each group. (C) Comparison of FWHM of the $v 1\left(\mathrm{PO}_{4}{ }^{3-}\right)$ peak in Raman spectra between each group. (D) Comparison of mineral-to-matrix ratio in Raman spectra between each group. (E) Representative FTIR spectra of bone close to the defect area in the PC, NC-2m, Mg-2m, NC-4m, and Mg-4m groups. (F) The differences in AUC of FTIR wavelengths between 1,200-1,750 $\mathrm{cm}^{-1}$ between each group normalized to that of healthy bone. (G) The proportion of bone mineral residue after heating to $700{ }^{\circ} \mathrm{C}$ for thermogravimetric analysis of dry weights. ${ }^{*} \mathrm{P}<0.05 ;{ }^{* * *} \mathrm{P}<0.01$.

\section{Bone recovery delayed by magnesium}

Changes that occurred in and around the defect area of the bone at 2 and 4 months after magnesium rod implantation were analyzed by micro-CT (Figure $5 A$ ).

The bone density and relative bone volume in the $\mathrm{Mg}$ group within a $5 \mathrm{~mm}$ diameter centered on the defect area were significantly inhibited compared to the NC group $(\mathrm{P}<0.05)$. Other cancellous bone parameters, including Tb.N and Tb.Sp, were also inhibited $(\mathrm{P}<0.05)$. The bone density, bone volume, and cancellous bone parameters had improved in each group by 4 months post-surgery; however, the inhibition evident in the $\mathrm{Mg}$ group had not significantly reversed. This same inhibition was also evident in the $1-\mathrm{mm}$ annular region outside the drilling area (Figure $5 B, C, D, E, F, G$ ).

\section{Histological evaluation of bone mineralization around magnesium rods}

The proportion of mineralized bone at the 1-mm annular region outside the drilling area in the $\mathrm{Mg}$ group was significantly lower than that in the NC group at 2 months post surgery $(6.1 \% \pm 1.1 \%$ vs. $24.0 \% \pm 4.9 \% ; \mathrm{P}<0.01)$. This difference remained at 4 months post surgery $(9.5 \% \pm 0.73 \%$ vs. $33.0 \% \pm 3.4 \% ; \mathrm{P}<0.01$ ) (Figure 6).

\section{Effect of magnesium rods on bone biomechanics}

The elastic modulus and maximum compressive force in both the $\mathrm{NC}$ and the $\mathrm{Mg}$ groups were significantly lower than those in the $\mathrm{PC}$ group at 2 months post surgery $(\mathrm{P}<0.01)$, but there was no significant difference between the $\mathrm{NC}$ and the $\mathrm{Mg}$ groups $(\mathrm{P}>0.05)$. By 4 months post surgery, the mechanical properties in the $\mathrm{NC}$ and $\mathrm{Mg}$ groups had improved. However, the elastic modulus and maximum compressive force in the $\mathrm{NC}$ group were significantly higher than those in the $\mathrm{Mg}$ group $(\mathrm{P}<0.05)$ (Figure 7).

\section{Discussion}

Many preclinical studies exploring magnesium and magnesium alloys for use as internal fixation devices have 

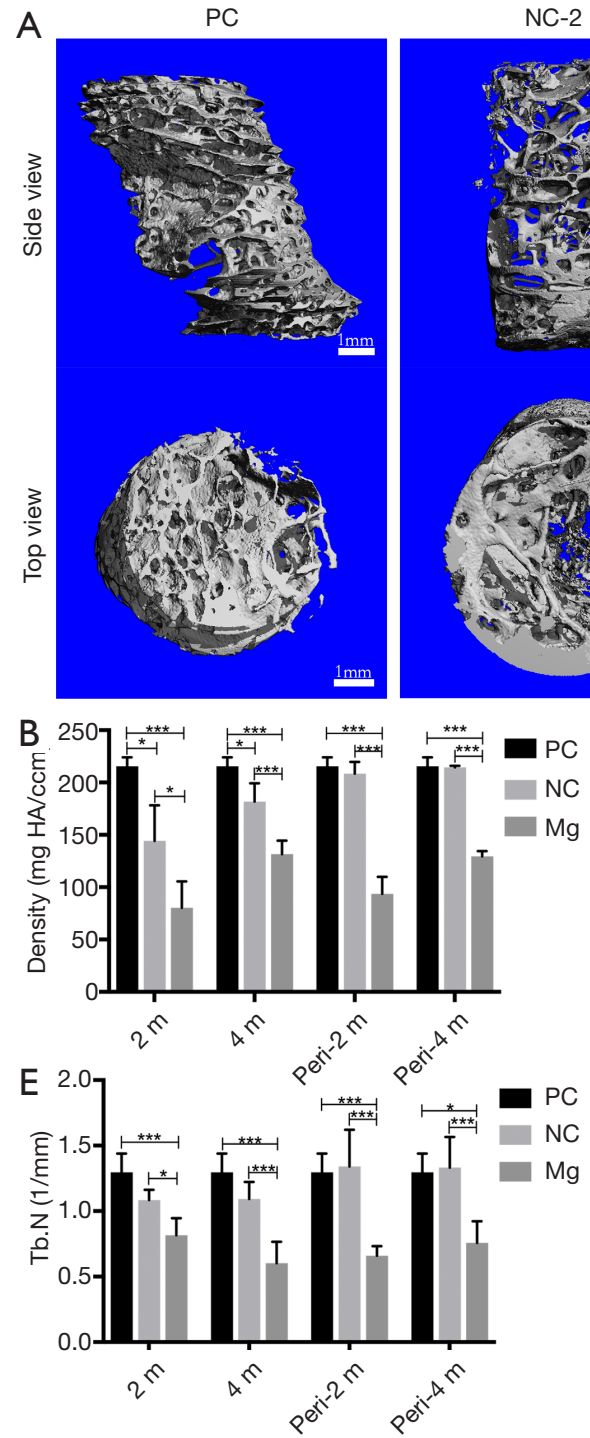

NC-2 m

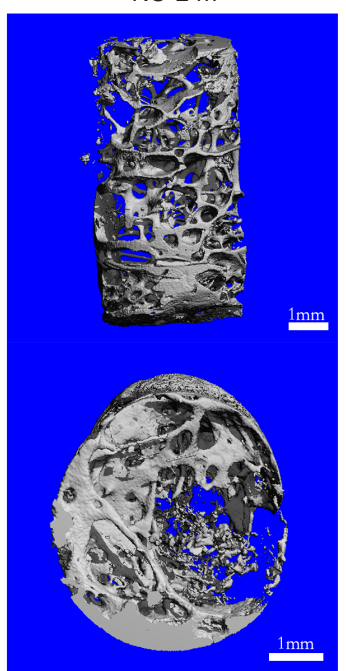

NC-4 m

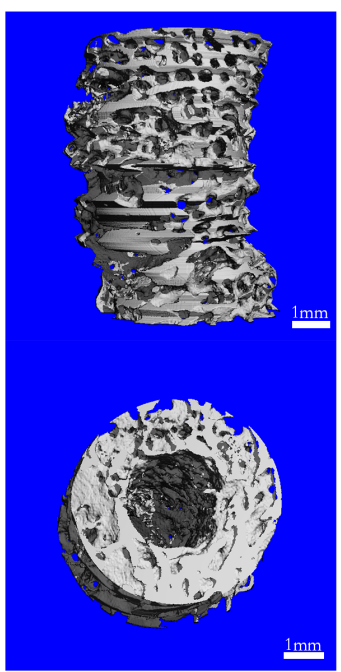

$\mathrm{Mg}-2 \mathrm{~m}$

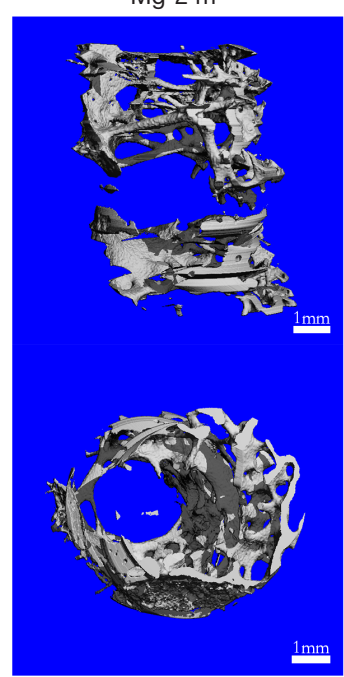

$\mathrm{Mg}-4 \mathrm{~m}$

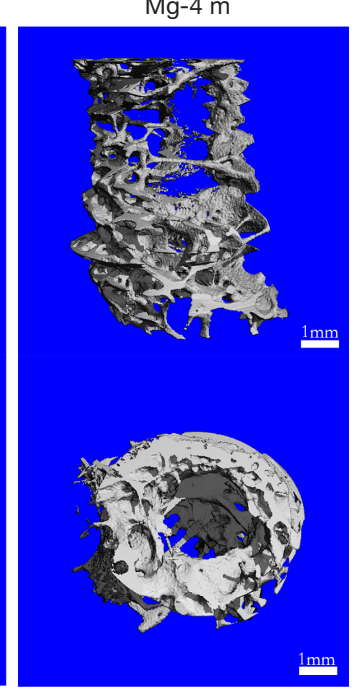

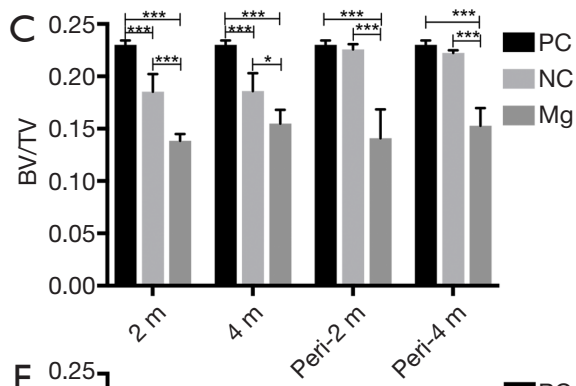
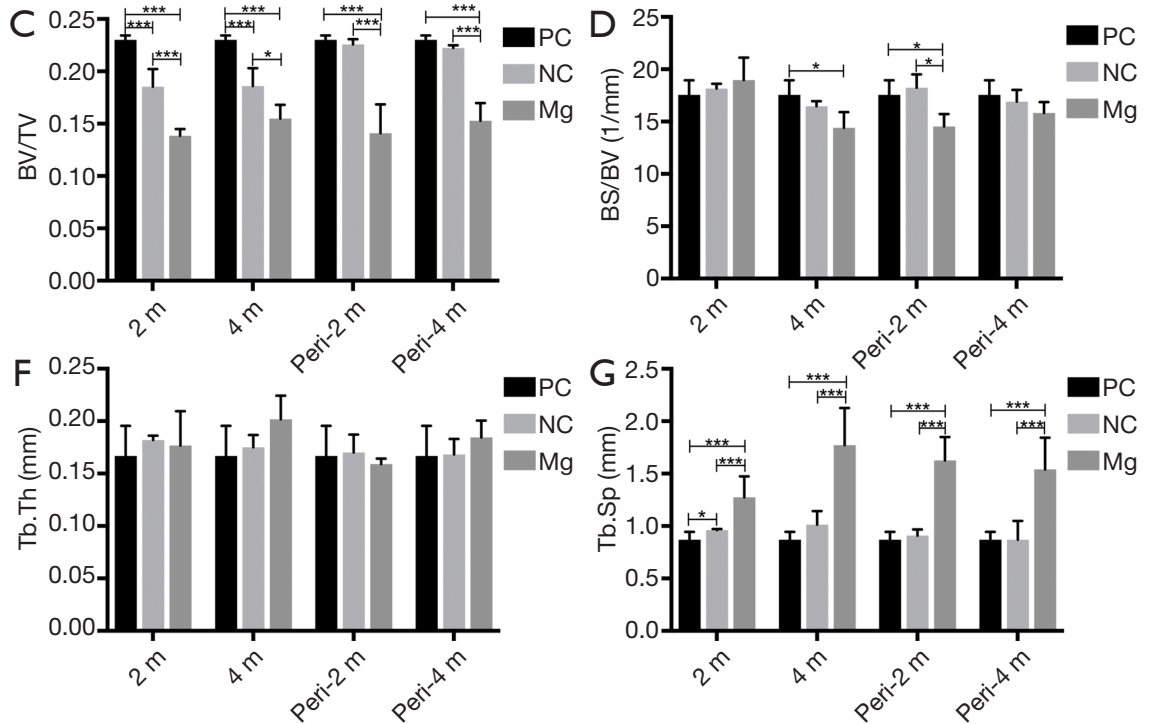

Figure 5 Histomorphometry analysis using micro-CT. (A) Micro-CT images of bone in the PC group, the NC-2m group, the NC-4m group, the $\mathrm{Mg}-2 \mathrm{~m}$ group, and the $\mathrm{Mg}-4 \mathrm{~m}$ group. (B,C,D,E,F,G) A comparison of histomorphometric parameters, including BMD, BV/TV, BS/BV, TB.N, Tb.Th, and Tb.Sp. $2 \mathrm{~m}$ and $4 \mathrm{~m}$ represent the bone parameter analysis within a $5 \mathrm{~mm}$ diameter centered on the drilling area at 2 and 4 months post surgery; peri- $2 \mathrm{~m}$ and peri- $4 \mathrm{~m}$ represent the bone parameter analysis of the 1 -mm annular column outside the drilling area at 2 and 4 months post-surgery. ${ }^{*} \mathrm{P}<0.05 ;{ }^{* * *} \mathrm{P}<0.01$.

focused on the effects magnesium has on bone healing and repair. However, few studies have considered the effects that magnesium implants have on bone quality. An understanding of bone quality is essential for a comprehensive understanding of bone biomechanics. Unlike other indicators of bone biomechanics, such as bone density and volume, bone quality addresses the composition and structure, both of which are related to bone mineral deposition (15). This study verified that exposure to high levels of magnesium disrupts bone mineralization both in vitro and in vivo, which alters bone properties. To facilitate the clinical transformation and promotion of magnesium and its alloys in orthopedics, we have formulated some suggestions based on our findings.

The biological effects of magnesium on bone can be observed to an extent in vitro, by examining the effect magnesium has on osteogenesis in MSCs. In this study, ALP expression increased in a magnesium ion concentration 

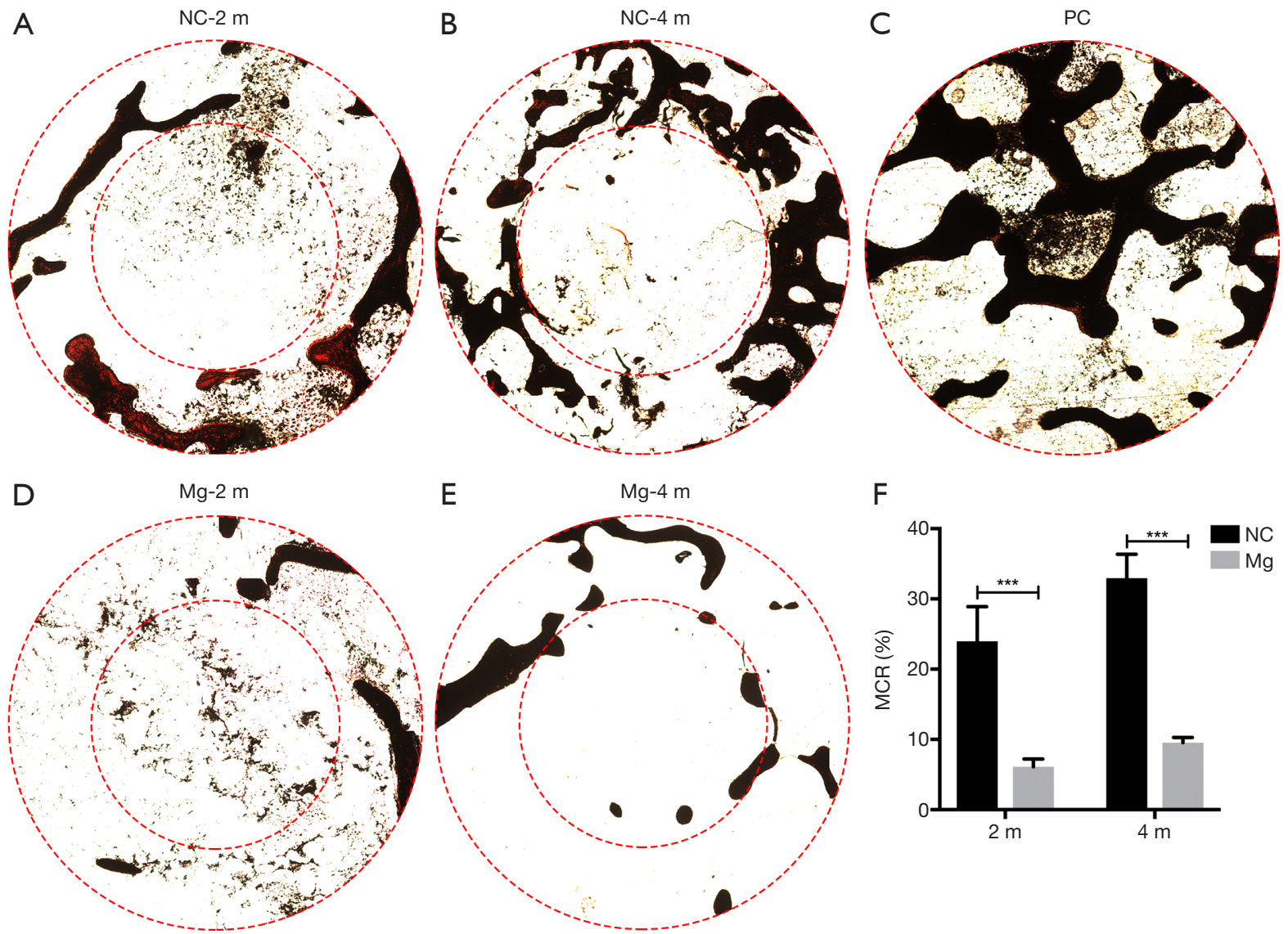

F

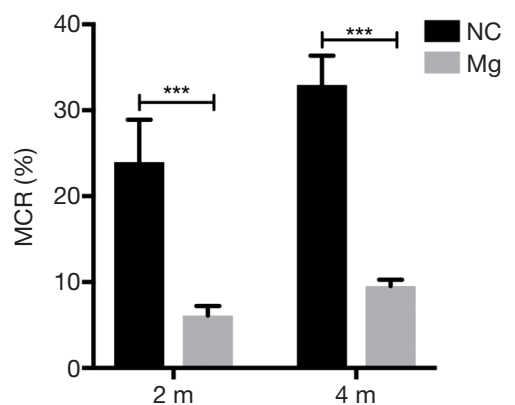

Figure 6 Mineralized bone with von Kossa and van Gieson staining to evaluate bone mineralization. Double staining of mineralized bone in the 1-mm annular column outside the drilling area in the following groups: (A) the NC-2m group; (B) the NC-4m group; (C) the PC group; (D) the $\mathrm{Mg}-2 \mathrm{~m}$ group; (E) and the $\mathrm{Mg}-4 \mathrm{~m}$ group. (F) The ratio of mineralized bone area to the $1-$ mm annular column area outside the drilling area (MCR) for each group. ${ }^{* * *} \mathrm{P}<0.01$.

gradient-dependent manner, which is consistent with several previous studies (20-22). This indicated that magnesium has a biological function in MSC osteogenesis. Interestingly, although we observed a continuous increase in ALP expression, bone mineral deposition was inhibited when exposed to high levels of magnesium. Yang et al. also observed this mineralization inhibition effect and attributed it to the inhibition of mitochondrial calcium ion potency, and to osteogenesis-related autophagy regulation $(23,24)$. In fact, ALP is only expressed during the early stages of osteogenically differentiated MSCs, and an increase in ALP expression does not necessarily indicate the formation of mature bone. Mature bone formation also requires bone mineral deposition in parallel with osteoblast differentiation and maturation. On the other hand, magnesium is an antagonist of calcium ions. Calcium- mediated mineralization in a high magnesium environment is therefore naturally competitively inhibited (25). Studies have shown that increases in serum magnesium concentration in patients with chronic renal failure or those who are undergoing dialysis are positively correlated with the patients' degree of bone mineralization defects (26). This also supports the notion that exposure to high levels of magnesium has a negative effect on bone mineralization.

Taking our in vitro findings into consideration, pure magnesium rods were implanted into the femoral condyle of rabbits and bone mineralization indicators were analyzed around the implantation region to further study the effects of magnesium on bone in vivo. In order to generate locally high levels of magnesium, we designed these magnesium rods with a porous structure to accelerate their degradation rate and reduce the dilution effect of the body's metabolism 

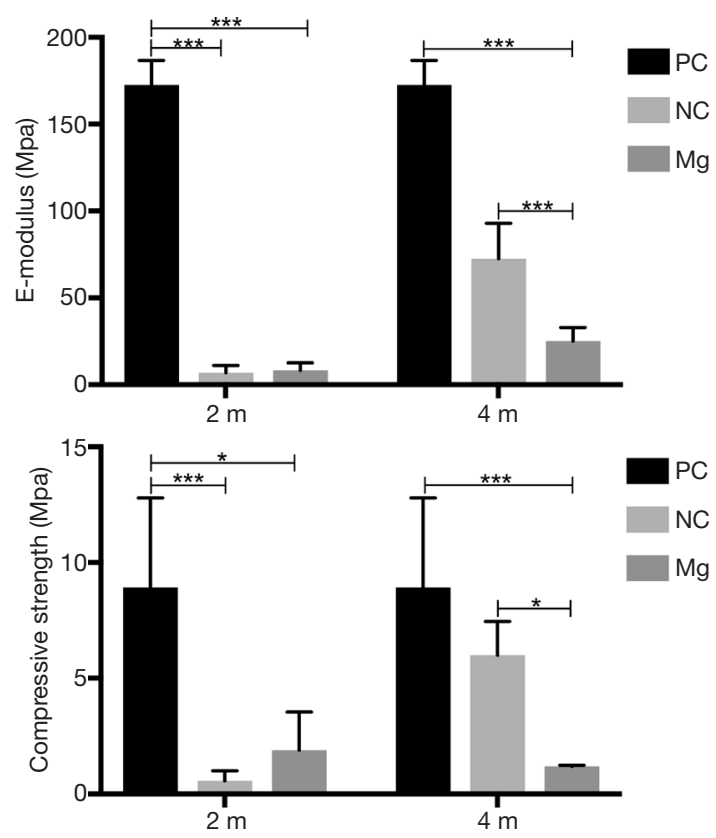

Figure 7 The effect of rapidly degrading magnesium rod implants on the biomechanics of bone. (A) A comparison of Young's modulus of bone in each group. (B) A comparison of the maximum compressive force of bone in each group. ${ }^{*} \mathrm{P}<0.05$; ${ }^{* *} \mathrm{P}<0.01$.

on local magnesium concentrations. EDS mapping showed a detectable distribution of magnesium within the bone in the vicinity of the implanted magnesium rods by 2 months post surgery. This suggests that exogenous magnesium produced by rod degradation can directly enter surrounding bone and play a biological role, and that this exogenous magnesium on adjacent bone can be cleared by the body once the rods have completely degraded. Calcium and phosphorus, according to our EDS results, play a more dominant role in the bone mineral phase.

Additional to the mineral phase, mineral crystallization and mineral-to-matrix ratio are important in maintaining healthy bone quality and ensuring normal bone function (27). Raman spectroscopy can be used to determine bone mineral composition and bone mineral crystallization $(28,29)$. The presence of a $v_{1}\left(\mathrm{PO}_{4}{ }^{3-}\right)$ phosphate peak close to $959 \mathrm{~cm}^{-1}$ on the Raman spectrum is an important bone marker (30). In this study, the $v_{1}\left(\mathrm{PO}_{4}{ }^{3-}\right)$ peak of healthy bone was close to $961 \mathrm{~cm}^{-1}$. For pure hydroxyapatite (HA), the wavelength position of the $v_{1}\left(\mathrm{PO}_{4}{ }^{3-}\right)$ peak has been reported at $964 \mathrm{~cm}^{-1}$. Any shift of this position suggests the formation of poorly crystalline $\mathrm{HA}$ or the presence of immature bone (31). The position of the $v_{1}\left(\mathrm{PO}_{4}{ }^{3-}\right)$ peak in the $\mathrm{Mg}$ and
NC groups was significantly different from the PC group at 2 months post surgery, suggesting the possibility of new HA formation during bone resorption and remodeling around the trauma site. However, the wavelength position of the $v_{1}\left(\mathrm{PO}_{4}{ }^{3-}\right)$ peak in the $\mathrm{Mg}$ group was significantly lower than that in the $\mathrm{NC}$ group by 4 months post surgery, which suggests delayed recovery of normal HA crystals in the $\mathrm{Mg}$ group. This possibly indicates that exogenous magnesium induced pathological HA crystallization or prolonged HA crystallization in bone peripheral to the drilled defect. The wavelength position of the $v_{1}\left(\mathrm{PO}_{4}{ }^{3-}\right)$ peak reflects quality of a crystal, whereas FWHM represents overall crystallinity of the bone tissue (30). A higher FWHM value suggests a lower degree of bone crystallinity, while a lower FWHM value indicates a higher degree of crystallinity $(29,31)$. In this study, consistent with previous reports, the FWHM for healthy bone was $16.7 \pm 0.6 \mathrm{~cm}^{-1}$. The FWHM value in the $\mathrm{Mg}$ and $\mathrm{NC}$ groups was significantly higher than that of healthy bone 2 months post surgery. However, the FWHM value in the $\mathrm{NC}$ group was not significantly different from that in healthy bone by 4 months post surgery, while the FWHM value in the $\mathrm{Mg}$ group was significantly higher than those in the $\mathrm{NC}$ and $\mathrm{PC}$ groups. One possible explanation is that, when crystallinity recovered in the $\mathrm{NC}$ group, the disruption to crystallization in the $\mathrm{Mg}$ group inhibited crystallinity for a longer period.

To explore the distribution of bone components, the ratio of $v_{1}\left(\mathrm{PO}_{4}^{-3}\right)$ peak intensity to type I collagen peak intensity in the Raman spectrum can be used to measure the mineralto-matrix ratio in bone (32). In this study, the mineral-tomatrix ratio in the $\mathrm{Mg}$ group was significantly lower than in the $\mathrm{NC}$ and $\mathrm{PC}$ groups at 2 months and 4 months post surgery, suggesting that bone mineral was less abundant than organic compounds in the $\mathrm{Mg}$ group. These results were partially verified by FTIR and thermogravimetric analyses. The spectra at wavelengths of 1,200 to $1,750 \mathrm{~cm}^{-1}$ as seen by FTIR primarily indicate matrix features (30). The ratio of AUC within this wavelength range relative to that in healthy bone was used to assess the relative content of organic compounds in bone samples. The relative matrix in the $\mathrm{NC}$ and $\mathrm{Mg}$ groups was significantly higher than that in healthy bone at 2 months post surgery, suggesting that bone remodeling post-trauma had occurred. The matrix in the NC group was similar to that in healthy bone by 4 months post surgery, but there was still no significant improvement seen in the $\mathrm{Mg}$ group, indicating that magnesium delayed the process of matrix resorption and mineralization during bone maturation. Our thermogravimetric results 
quantitatively reflected that bone mineral growth was significantly inhibited by rapidly degraded magnesium. It is worth noting that an alkaline environment, which can occur following magnesium degradation, is beneficial to mineral growth (33). The inhibition shown in this study, however, indicates that the inhibitory effect of a local magnesiumrich environment exceeds the promoting effects of a local alkaline environment.

Bone density and bone biomechanics are also affected by this mineral growth inhibition. Micro-CT analysis showed that bone density and volume in and around the drilling area were significantly inhibited by the rapid degradation of magnesium rods. The same effect could be seen in the cancellous bone parameters Tb.N and Tb.Sp. Dziuba et al. also found that pure magnesium nails implanted in the medullary cavities of rabbits decreased bone density and increased bone porosity of cortical bone surrounding the implants (12). Due to the porous structure of the magnesium rods used in the present study, they had faster degradation rates and had degraded almost completely by 2 months post surgery. This increased the inhibitory effect of the magnesium. Histologically, this led to a significantly reduced area of mineralized bone in the annular region surrounding the defect area following implantation, which confirmed the micro-CT findings. Based on this, it can be projected that the mechanical properties of bone were also inhibited.

While it is true that magnesium stimulates bone growth to an extent, this disruption of bone mineralization caused by a local high magnesium environment cannot be ignored. Based on our findings, we can provide several suggestions which may be helpful in developing rational clinical applications of magnesium and its alloys. First, it is essential to control magnesium concentrations in the implant location to avoid mineralization disruption, primarily during fracture healing. From this perspective, magnesium products with slower rates of degradation may be more effective and safer in clinical application. Second, close monitoring of magnesium-based implants is required, as well as secondary fracture prevention. Secondary fractures may cause decreased bone strength and an increased risk of re-fracture. Specifically, diligent assessment by X-ray or CT should be provided to patients and, in cases where bone mineralization inhibition is discovered, advice provided as to the prevention of further fractures. Third, magnesiumbased devices are more suitable at specific sites. According to various preclinical studies, almost all the new bone that grows around degraded magnesium products does so beneath the periosteum $(5,10,11,34)$. In our opinion, the plentiful blood supply and loose tissue conditions at this site allow degraded magnesium to be rapidly metabolized. This reduces magnesium accumulation and any subsequent inhibitory effects on bone mineral deposition. Conversely, magnesium accumulation is likely to occur in the relatively closed environment within a bone defect. This means that magnesium-based products appear to be unsuitable as bone filler blocks. However, a sustained release system using magnesium ions or small amounts of granular magnesium as an osteogenic factor could be a feasible method to promote bone defect repair $(35,36)$.

\section{Conclusions}

In this study, we observed that high levels of magnesium can stimulate the early osteogenic differentiation of hBMSCs in vitro, but inhibit MSC-mediated late mineralization. In vivo experiments confirmed that exposure to high levels of magnesium disrupts mineralization activities, such as bone mineral crystallization and the mineral to matrix balance, reducing bone quality and biomechanical function. Our study results have the potential to guide the refinement of magnesium-based internal fixation devices to the point where they are ready for widespread clinical use.

\section{Acknowledgments}

All authors sincerely thank all volunteers for their support for this work.

Funding: This work was supported by the Shanghai Jiao Tong University Medical and Engineering Cross Fund (Grant No. YG2015QN41), the Shanghai Clinical Medical Center (Grant Number 2017ZZ01023), the Shanghai Municipal Key Clinical Specialty, the National Nature Science Foundation of China (Grant No.81902195) and China Postdoctoral Science Foundation (2018M640406).

\section{Footnote}

Reporting Checklist: The authors present the study in accordance with the ARRIVE reporting checklist. Available at: http://dx.doi.org/10.21037/atm-20-1921

Data Sharing Statement: Available at http://dx.doi. org/10.21037/atm-20-1921

Conflicts of Interest: All authors have completed the ICMJE 
uniform disclosure form (available at http://dx.doi. org/10.21037/atm-20-1921). The authors have no conflicts of interest to declare.

Ethical Statement: The authors are accountable for all aspects of the work in ensuring that questions related to the accuracy or integrity of any part of the work are appropriately investigated and resolved. Animal experiments were performed under a project license (HKDL[2018]293) granted by the Animal Ethics Committee of the Shanghai Ninth People's Hospital affiliated with the Shanghai Jiao Tong University School of Medicine, in compliance with Shanghai Ninth People's Hospital affiliated with the Shanghai Jiao Tong University School of Medicine guidelines for the care and use of animals..

Open Access Statement: This is an Open Access article distributed in accordance with the Creative Commons Attribution-NonCommercial-NoDerivs 4.0 International License (CC BY-NC-ND 4.0), which permits the noncommercial replication and distribution of the article with the strict proviso that no changes or edits are made and the original work is properly cited (including links to both the formal publication through the relevant DOI and the license). See: https://creativecommons.org/licenses/by-nc-nd/4.0/.

\section{References}

1. Zhao D, Witte F, Lu F, et al. Current status on clinical applications of magnesium-based orthopaedic implants: A review from clinical translational perspective. Biomaterials 2017;112:287-302.

2. Claes LE. Mechanical characterization of biodegradable implants. Clin Mater 1992;10:41-6.

3. Kannan MB, Raman RK. In vitro degradation and mechanical integrity of calcium-containing magnesium alloys in modified-simulated body fluid. Biomaterials 2008;29:2306-14.

4. Staiger MP, Pietak AM, Huadmai J, et al. Magnesium and its alloys as orthopedic biomaterials: a review. Biomaterials 2006;27:1728-34.

5. Chaya A, Yoshizawa S, Verdelis K, et al. In vivo study of magnesium plate and screw degradation and bone fracture healing. Acta Biomater 2015;18:262-9.

6. Han $\mathrm{P}$, Cheng $\mathrm{P}$, Zhang $\mathrm{S}$, et al. In vitro and in vivo studies on the degradation of high-purity $\mathrm{Mg}(99.99 \mathrm{wt} . \%)$ screw with femoral intracondylar fractured rabbit model. Biomaterials 2015;64:57-69.
7. Wu L, Feyerabend F, Schilling AF, et al. Effects of extracellular magnesium extract on the proliferation and differentiation of human osteoblasts and osteoclasts in coculture. Acta Biomater 2015;27:294-304.

8. Luthringer BJ, Willumeit-Romer R. Effects of magnesium degradation products on mesenchymal stem cell fate and osteoblastogenesis. Gene 2016;575:9-20.

9. Yang C, Yuan G, Zhang J, et al. Effects of magnesium alloys extracts on adult human bone marrow-derived stromal cell viability and osteogenic differentiation. Biomed Mater 2010;5:045005.

10. Chaya A, Yoshizawa S, Verdelis K, et al. Fracture healing using degradable magnesium fixation plates and screws. J Oral Maxillofac Surg 2015;73:295-305.

11. Henderson SE, Verdelis K, Maiti S, et al. Magnesium alloys as a biomaterial for degradable craniofacial screws. Acta Biomater 2014;10:2323-32.

12. Dziuba D, Meyer-Lindenberg A, Seitz JM, et al. Longterm in vivo degradation behaviour and biocompatibility of the magnesium alloy ZEK100 for use as a biodegradable bone implant. Acta Biomater 2013;9:8548-60.

13. Lee JW, Han HS, Han KJ, et al. Long-term clinical study and multiscale analysis of in vivo biodegradation mechanism of $\mathrm{Mg}$ alloy. Proc Natl Acad Sci U S A 2016;113:716-21.

14. Kim YK, Lee KB, Kim SY, et al. Gas formation and biological effects of biodegradable magnesium in a preclinical and clinical observation. Sci Technol Adv Mater 2018;19:324-35.

15. Seeman E, Delmas PD. Bone quality--the material and structural basis of bone strength and fragility. $\mathrm{N} \mathrm{Engl} \mathrm{J}$ Med 2006;354:2250-61.

16. Mori S. Contribution of bone quality to fracture risk. Clin Calcium 2004;14:33-8.

17. Fyhrie DP. Summary--Measuring "bone quality". J Musculoskelet Neuronal Interact 2005;5:318-20.

18. Chu W, Zhuang Y, Gan Y, et al. Comparison and characterization of enriched mesenchymal stem cells obtained by the repeated filtration of autologous bone marrow through porous biomaterials. J Transl Med 2019;17:377.

19. Jia GZ, Hou Y, Chen CX, et al. Precise fabrication of open porous $\mathrm{Mg}$ scaffolds using $\mathrm{NaCl}$ templates: Relationship between space holder particles, pore characteristics and mechanical behavior. Materials \& Design 2018;140:106-13.

20. Leem YH, Lee KS, Kim JH, et al. Magnesium ions facilitate integrin alpha 2-and alpha 3-mediated proliferation and enhance alkaline phosphatase expression 
and activity in hBMSCs. J Tissue Eng Regen Med 2016;10:E527-36.

21. Kim BS, Kim JS, Park YM, et al. Mg ion implantation on SLA-treated titanium surface and its effects on the behavior of mesenchymal stem cell. Mater Sci Eng C Mater Biol Appl 2013;33:1554-60.

22. Lin S, Yang G, Jiang F, et al. A Magnesium-Enriched 3D Culture System that Mimics the Bone Development Microenvironment for Vascularized Bone Regeneration. Adv Sci (Weinh) 2019;6:1900209.

23. Zhang L, Yang C, Li J, et al. High extracellular magnesium inhibits mineralized matrix deposition and modulates intracellular calcium signaling in human bone marrowderived mesenchymal stem cells. Biochem Biophys Res Commun 2014;450:1390-5.

24. Li Y, Wang J, Yue J, et al. High magnesium prevents matrix vesicle-mediated mineralization in human bone marrow-derived mesenchymal stem cells via mitochondrial pathway and autophagy. Cell Biol Int 2018;42:205-15.

25. Hamano N, Komaba H, Fukagawa M. Magnesium as a new player in CKD: too little is as bad as too much? Kidney Int 2017;92:1034-6.

26. Castiglioni S, Cazzaniga A, Albisetti W, et al. Magnesium and osteoporosis: current state of knowledge and future research directions. Nutrients 2013;5:3022-33.

27. Addison WN, Nelea V, Chicatun F, et al. Extracellular matrix mineralization in murine MC3T3-E1 osteoblast cultures: an ultrastructural, compositional and comparative analysis with mouse bone. Bone 2015;71:244-56.

28. McManus LL, Burke GA, McCafferty MM, et al.

Cite this article as: Chu W, Li T, Jia G, Chang Y, Liu Z, Pei J, Yu D, Zhai Z. Exposure to high levels of magnesium disrupts bone mineralization in vitro and in vivo. Ann Transl Med 2020;8(21):1419. doi: 10.21037/atm-20-1921
Raman spectroscopic monitoring of the osteogenic differentiation of human mesenchymal stem cells. Analyst 2011;136:2471-81.

29. Mandair GS, Morris MD. Contributions of Raman spectroscopy to the understanding of bone strength. Bonekey Rep 2015;4:620.

30. Morris MD, Mandair GS. Raman assessment of bone quality. Clin Orthop Relat Res 2011;469:2160-9.

31. Alakpa EV, Burgess KEV, Chung P, et al. Nacre Topography Produces Higher Crystallinity in Bone than Chemically Induced Osteogenesis. ACS Nano 2017;11:6717-27.

32. McCreadie BR, Morris MD, Chen TC, et al. Bone tissue compositional differences in women with and without osteoporotic fracture. Bone 2006;39:1190-5.

33. Marelli B, Ghezzi CE, Zhang YL, et al. Fibril formation $\mathrm{pH}$ controls intrafibrillar collagen biomineralization in vitro and in vivo. Biomaterials 2015;37:252-9.

34. Yang JX, Cui FZ, Lee IS, et al. In vivo biocompatibility and degradation behavior of $\mathrm{Mg}$ alloy coated by calcium phosphate in a rabbit model. J Biomater Appl 2012;27:153-64.

35. Lai Y, Li Y, Cao H, et al. Osteogenic magnesium incorporated into PLGA/TCP porous scaffold by $3 \mathrm{D}$ printing for repairing challenging bone defect. Biomaterials 2019;197:207-19.

36. Zhang W, Feng C, Yang G, et al. 3D-printed scaffolds with synergistic effect of hollow-pipe structure and bioactive ions for vascularized bone regeneration. Biomaterials 2017;135:85-95. 